УДК 517.9

\title{
An Identification Problem of Nonlinear Lowest Term Coefficient in the Special Form for Two-Dimensional Semilinear Parabolic Equation
}

\author{
Ekaterina N. Kriger* \\ Igor V. Frolenkov ${ }^{\dagger}$ \\ Institute of Mathematics and Computer Science \\ Siberian Federal University \\ Svobodny, 79, Krasnoyarsk, 660041
}

Russia

Received 10.12.2015, received in revised form 16.02.2016, accepted 18.03.2016

In this paper we investigate an identification problem of a coefficient at the nonlinear lowest term in a $2 D$ semilinear parabolic equation with overdetermination conditions given on a smooth curve. The unknown coefficient has the form of a product of two functions each depending on time and a spatial variable. We prove solvability of the problem in classes of smooth bounded functions. We present an example of input data satisfying the conditions of the theorem and the corresponding solution.

Keywords: inverse problem, semilinear parabolic equation, Cauchy problem, lowest term coefficient, weak approximation method, local solvability, overdetermination conditions on a smooth curve.

DOI: 10.17516/1997-1397-2016-9-2-180-191.

We consider an identification problem of a special coefficient at the nonlinear term in a twodimensional semilinear parabolic equation with Cauchy data. The desired coefficient depends on all variables and has the form of a product of two functions each depending on time and a spatial variable. Overdetermination conditions are given on two smooth curves.

By means of overdetermination conditions the given inverse problem is reduced to a nonclassical direct problem for a loaded parabolic equation. Solvability of the direct problem is proved by the weak approximation method [1-3]. Efficiency of the weak approximation method (the splitting method at differential level) is that, as a rule, on every fractional step the split problem is simpler than the original one. Therefore we can estimate the solution of the split problem more precisely, obtain a priori estimates and establish its solvability.

For the original inverse problem we prove a theorem of the solution existence in classes of smooth bounded functions.

Earlier, the problems of coefficient identification for semilinear parabolic equations with Cauchy data have been studied in papers $[4,5]$. In [6] the problem of identification of two coefficients in a semilinear parabolic equation with overdetermination conditions on smooth curves has been considered. Solvability of the identification problem of a coefficient represented as a sum of two functions at a nonlinear term in a semilinear parabolic equation has been investigated in [7].

In paper [8] unique solvability of an inverse boundary-value problem for an one-dimensional semilinear parabolic equation with the sought for coefficient $f(t)+g(x)$ at the lowest term has

\footnotetext{
*e_katherina@mail.ru

†igor@frolenkov.ru

(c) Siberian Federal University. All rights reserved
} 
been proved. The boundary-value problem of identification of the coefficient at $u(t, x)$ depending on $t, x$ in a parabolic equation has been considered in [9].

In [10] we proved existence and uniqueness of solution of the identification problem of the coefficient $\lambda(t, x, z)=\lambda_{1}(t, x) \cdot \lambda_{2}(t, z)$ at the source function in a two-dimensional parabolic equation with Cauchy data. The identification problems of coefficients of other forms at the source function in parabolic equations have been studied in papers $[11,12]$.

\section{The problem definition}

In the strip $G_{[0, T]}=\left\{(t, x, z) \mid 0 \leqslant t \leqslant T,(x, z) \in \mathbb{R}^{2}\right\}$ we consider a Cauchy problem for the parabolic equation

$$
\begin{gathered}
u_{t}=L(u(t, x, z))+u^{p} \cdot \lambda(t, x, z)+f(t, x, z), \quad(t, x, z) \in G_{(0, T]}, \\
L(u(t, x, z))=\alpha_{1}(t) \cdot u_{x x}(t, x, z)+\alpha_{2}(t) \cdot u_{z z}(t, x, z)+\beta_{1}(t) \cdot u_{x}(t, x, z)+\beta_{2}(t) \cdot u_{z}(t, x, z),
\end{gathered}
$$

with the initial condition

$$
u(0, x, z)=u_{0}(x, z),(x, z) \in \mathbb{R}^{2} .
$$

Here the degree $p \geqslant 1$ is an integer constant. The coefficients $\alpha_{k}(t) \geqslant \alpha_{0}>0, \beta_{k}(t), k=1,2$, are real-valued, continuous and bounded in $[0, T]$ functions.

Together with the function $u(t, x, z)$ we also need to determine the function

$$
\lambda(t, x, z)=\lambda_{1}(t, x) \cdot \lambda_{2}(t, z) .
$$

Let the function $u(t, x, z)$ satisfies overdetermination conditions

$$
u(t, x, a(t))=\varphi(t, x), u(t, b(t), z)=\psi(t, z),
$$

where the curves $a(t), b(t) \in C^{1}([0, T])$.

Suppose the compatibility conditions are fulfilled

$$
u_{0}(x, a(0))=\varphi(0, x), \quad u_{0}(b(0), z)=\psi(0, z), \quad \varphi(t, b(t))=\psi(t, a(t)) .
$$

Assume also that the $f(t, x, z), u_{0}(x, z), \varphi(t, x), \psi(t, z)$ defined in $G_{[0, T]}, \mathbb{R}^{2},[0, T] \times \mathbb{R}$, $[0, T] \times \mathbb{R}$, respectively, are continuous, have continuous derivatives occurring in the relation $(7)$ and satisfy it:

$$
\begin{aligned}
\left|\frac{\partial^{l_{1}}}{\partial t^{l_{1}}} \frac{\partial^{k_{1}}}{\partial x^{k_{1}}} \varphi(t, x)\right|+\left|\frac{\partial^{l_{2}}}{\partial t^{l_{2}}} \frac{\partial^{k_{2}}}{\partial z^{k_{2}}} \psi(t, z)\right|+\left|\frac{\partial^{k_{1}}}{\partial x^{k_{1}}} \frac{\partial^{k_{2}}}{\partial z^{k_{2}}} u_{0}(x, z)\right|+ \\
\quad+\left|\frac{\partial^{k_{1}}}{\partial x^{k_{1}}} \frac{\partial^{k_{2}}}{\partial z^{k_{2}}} f(t, x, z)\right| \leqslant C, l_{1}, l_{2}=0,1, k_{1}, k_{2}=0,1, \ldots, 6 .
\end{aligned}
$$

Let also the following constraints on the initial data hold

$$
\begin{gathered}
|\varphi(t, x)| \geqslant \delta_{1}>0, \quad|\psi(t, z)| \geqslant \delta_{2}>0, \quad \forall(t, x, z) \in G_{[0, T]}, \\
\mid \varphi_{t}(t, b(t))-\alpha_{1}(t) \cdot \varphi_{x x}(t, b(t))-\alpha_{2}(t) \cdot \psi_{z z}(t, a(t))-\beta_{1}(t) \cdot \varphi_{x}(t, b(t))- \\
-\beta_{2}(t) \cdot \psi_{z}(t, a(t)) \cdot\left(1+a^{\prime}(t)\right)-f(t, b(t), a(t)) \mid \geqslant \delta_{3}, \forall t \in[0, T],
\end{gathered}
$$

where $\delta_{1}, \delta_{2}, \delta_{3}$ are constants. 


\section{Reduction to the direct problem}

Let us reduce the inverse problem (1)-(5) to the auxiliary direct problem. For this purpose in equation (1) we set $x=b(t)$ to express the coefficient $\lambda_{1}(t, b(t)) \cdot \lambda_{2}(t, z)$. After that in (1) we set $z=a(t)$ to express the coefficient $\lambda_{1}(t, x) \cdot \lambda_{2}(t, a(t))$. In the product of these two expressions we substitute the expression for $\lambda_{1}(t, b(t)) \cdot \lambda_{2}(t, a(t))$ obtained from (1) by simultaneous substitution of $x=b(t), z=a(t)$.

By (5) we obtain the following expression for the unknown coefficient:

$$
\begin{array}{r}
\lambda(t, x, z)=\frac{\varphi^{p}(t, b(t))}{æ(t)} \cdot\left(\frac{\varphi_{t}(t, x)-\alpha_{1}(t) \varphi_{x x}(t, x)-\beta_{1}(t) \varphi_{x}(t, x)-f(t, x, a(t))}{\varphi^{p}(t, x)}-\right. \\
\left.-\frac{\alpha_{2}(t) u_{z z}(t, x, a(t))+\beta_{2}(t) u_{z}(t, x, a(t)) \cdot\left(1+a^{\prime}(t)\right)}{\varphi^{p}(t, x)}\right) \cdot\left(\frac{\psi_{t}(t, z)-\alpha_{2}(t) \psi_{z z}(t, z)}{\psi^{p}(t, z)}-\right. \\
\left.-\frac{\beta_{2}(t) \psi_{z}(t, z)+f(t, b(t), z)}{\psi^{p}(t, z)}-\frac{\alpha_{1}(t) u_{x x}(t, b(t), z)+\beta_{1}(t) u_{x}(t, b(t), z) \cdot\left(1+b^{\prime}(t)\right)}{\psi^{p}(t, z)}\right),
\end{array}
$$

where

$$
\begin{aligned}
æ(t)=\varphi_{t}(t, b(t))-\alpha_{1}(t) \cdot \varphi_{x x}(t, b(t))-\alpha_{2}(t) & \cdot \psi_{z z}(t, a(t))-\beta_{1}(t) \cdot \varphi_{x}(t, b(t))- \\
& -\beta_{2}(t) \cdot \psi_{z}(t, a(t)) \cdot\left(1+a^{\prime}(t)\right)-f(t, b(t), a(t)) .
\end{aligned}
$$

We transform relation (10) to the form

$$
\begin{aligned}
\lambda(t, x, z)=A_{0}(t) \cdot\left(A_{1}(t, x)+A_{2}(t, x) \cdot u_{z z}(t, x, a(t))+A_{3}(t, x) \cdot u_{z}(t, x, a(t))\right) \times \\
\times\left(B_{1}(t, z)+B_{2}(t, z) \cdot u_{x x}(t, b(t), z)+B_{3}(t, z) \cdot u_{x}(t, b(t), z)\right),
\end{aligned}
$$

where the functions $A_{0}(t), A_{i}(t, x), B_{i}(t, z), i=1,2,3$, are known, depend on the initial data and given by the following formulas:

$$
\begin{aligned}
& A_{0}(t)=\frac{\varphi^{p}(t, b(t))}{x(t)} ; \quad A_{1}(t, x)=\frac{\varphi_{t}(t, x)-\alpha_{1}(t) \varphi_{x x}(t, x)-\beta_{1}(t) \varphi_{x}(t, x)-f(t, x, a(t))}{\varphi^{p}(t, x)} ; \\
& A_{2}(t, x)=-\frac{\alpha_{2}(t)}{\varphi^{p}(t, x)} ; \quad B_{1}(t, z)=\frac{\psi_{t}(t, z)-\alpha_{2}(t) \psi_{z z}(t, z)-\beta_{2}(t) \psi_{z}(t, z)-f(t, b(t), z)}{\psi^{p}(t, z)} ; \\
& A_{3}(t, x)=-\frac{\beta_{2}(t) \cdot\left(1+a^{\prime}(t)\right)}{\varphi^{p}(t, x)} ; \quad B_{2}(t, z)=-\frac{\alpha_{1}(t)}{\psi^{p}(t, z)} ; \quad B_{3}(t, z)=-\frac{\beta_{1}(t) \cdot\left(1+b^{\prime}(t)\right)}{\psi^{p}(t, z)}
\end{aligned}
$$

Substituting the relation (11) to equation (1) we arrive at the direct problem for the equation

$$
u_{t}(t, x, z)=L(u(t, x, z))+\lambda(t, x, z) \cdot u^{p}(t, x, z)+f(t, x, z),(t, x, z) \in G_{(0, T]},
$$

with the initial condition (3) and the coefficient $\lambda(t, x, z)$ of the form (11).

\section{Existence of solution of the direct problem}

To prove solvability of the direct problem (13), (3) we use the weak approximation method [1-3]. We split equation (13) into two fractional steps and make a shift by $\frac{\tau}{2}$ in nonlinear terms and traces of unknown functions.

$$
u_{t}^{\tau}=2 \cdot L\left(u^{\tau}(t, x, z)\right), n \tau<t \leqslant\left(n+\frac{1}{2}\right) \tau ;
$$




$$
\begin{gathered}
u_{t}^{\tau}=2 \cdot\left(u^{\tau}\left(t-\frac{\tau}{2}, x, z\right)\right)^{p} \cdot M^{\tau}\left(t, x, z, u^{\tau}\left(t-\frac{\tau}{2}, x, z\right)\right)+2 \cdot f(t, x, z),\left(n+\frac{1}{2}\right) \tau<t \leqslant(n+1) \tau \\
u^{\tau}(0, x, z)=u_{0}(x, z) \\
n=0,1,2, \ldots,(N-1), N \tau=T .
\end{gathered}
$$

Here the coefficient at the nonlinear term has the following form:

$$
\begin{gathered}
M^{\tau}\left(t, x, z, u^{\tau}\left(t-\frac{\tau}{2}, x, z\right)\right)=\left(B_{1}(t, z)+B_{2}(t, z) \cdot u_{x x}^{\tau}\left(t-\frac{\tau}{2}, b(t), z\right)+B_{3}(t, z) \cdot u_{x}^{\tau}\left(t-\frac{\tau}{2}, b(t), z\right)\right) \times \\
\times\left(A_{1}(t, x)+A_{2}(t, x) \cdot u_{z z}^{\tau}\left(t-\frac{\tau}{2}, x, a(t)\right)+A_{3}(t, x) \cdot u_{z}^{\tau}\left(t-\frac{\tau}{2}, x, a(t)\right)\right) \cdot A_{0}(t) . \quad(17)
\end{gathered}
$$

By the $n$-th complete step we mean the half-interval $(n \tau,(n+1) \tau]$, whereas the $j$-th fractional step of the $n$-th complete step is the half-interval $((n+(j-1) / 2) \tau,(n+j / 2) \tau]$.

Let us introduce the notation

$$
\begin{aligned}
& U_{k_{1}, k_{2}}(0)=\sup _{(x, z) \in \mathbb{R}^{2}}\left|\frac{\partial^{k_{1}}}{\partial x^{k_{1}}} \frac{\partial^{k_{2}}}{\partial z^{k_{2}}} u_{0}(x, z)\right| \\
& U_{k_{1}, k_{2}}^{\tau}(t)=\sup _{n \tau<\xi \leqslant t} \sup _{(x, z) \in \mathbb{R}^{2}}\left|\frac{\partial^{k_{1}}}{\partial x^{k_{1}}} \frac{\partial^{k_{2}}}{\partial z^{k_{2}}} u^{\tau}(\xi, x, z)\right|, \quad n \tau<t \leqslant(n+1) \tau, \\
& k_{1}, k_{2}=0,1, \ldots, 6, \\
& U(0)=\sum_{k_{1}=0}^{6} \sum_{k_{2}=0}^{6} U_{k_{1}, k_{2}}(0), \quad U^{\tau}(t)=\sum_{k_{1}=0}^{6} \sum_{k_{2}=0}^{6} U_{k_{1}, k_{2}}^{\tau}(t) .
\end{aligned}
$$

The following statements are true

1. $\left|\frac{\partial^{k_{1}}}{\partial x^{k_{1}}} \frac{\partial^{k_{2}}}{\partial z^{k_{2}}} u^{\tau}(\xi, x, z)\right| \leqslant U_{k_{1}, k_{2}}^{\tau}(t) \leqslant U^{\tau}(t), k_{1}, k_{2}=0,1, \ldots, 6$,

$$
n \tau<\xi \leqslant t, n \tau<t \leqslant(n+1) \tau
$$

2. The functions $U_{k_{1}, k_{2}}^{\tau}(t), U^{\tau}(t)$ are nonnegative and nondecreasing on each time step $(n \tau,(n+1) \tau]$.

Let us prove a priori estimates guaranteeing the compactness of the family of solutions $u^{\tau}(t, x, z)$ of the problem $(14)-(16)$.

We consider the zeroth complete step $(n=0)$. At the first fractional step with $t \in\left(0, \frac{\tau}{2}\right]$ we study the equation

$$
u_{t}^{\tau}=2 \cdot L\left(u^{\tau}(t, x, z)\right)
$$

with initial condition (16).

By the maximum principle for the Cauchy problem (14), (16) we find

$$
\left|u^{\tau}(\xi, x, z)\right| \leqslant \sup _{(x, z) \in \mathbb{R}^{2}}\left|u_{0}(x, z)\right|, \quad 0<\xi \leqslant t, \quad t \in\left(0, \frac{\tau}{2}\right] .
$$

Differentiating equation (14) and the initial condition (16) with respect to $x$ and $z$ from 1 to 6 times and using the maximum principle we obtain

$$
\begin{aligned}
\left|\frac{\partial^{k_{1}}}{\partial x^{k_{1}}} \frac{\partial^{k_{2}}}{\partial z^{k_{2}}} u^{\tau}(\xi, x, z)\right| \leqslant \sup _{(x, z) \in \mathbb{R}^{2}}\left|\frac{\partial^{k_{1}}}{\partial x^{k_{1}}} \frac{\partial^{k_{2}}}{\partial z^{k_{2}}} u_{0}(x, z)\right|, & \\
& 0<\xi \leqslant t, \quad t \in\left(0, \frac{\tau}{2}\right], \quad k_{1}, k_{2}=0,1, \ldots, 6 .
\end{aligned}
$$


We apply $\sup _{(x, z) \in \mathbb{R}^{2}}$ first to the right parts of the inequalities $(20),(21)$ and then to their left parts. After that we apply $\sup _{0<\xi \leqslant t}$ to both sides of the obtained relations and sum up the results. In view of (18) and (19) we get

$$
U^{\tau}(t) \leqslant U(0), \quad t \in\left(0, \frac{\tau}{2}\right]
$$

In the second fractional step $\left(t \in\left(\frac{\tau}{2}, \tau\right]\right)$ we have

$$
\begin{gathered}
u_{t}^{\tau}=2 \cdot\left(u^{\tau}\left(t-\frac{\tau}{2}, x, z\right)\right)^{p} \cdot M^{\tau}\left(t, x, z, u^{\tau}\left(t-\frac{\tau}{2}, x, z\right)\right)+2 \cdot f(t, x, z), \\
\left.u^{\tau}\right|_{t-\frac{\tau}{2}}=u^{\tau}\left(\frac{\tau}{2}, x, z\right) .
\end{gathered}
$$

Then we integrate equation (15) with respect to the time variable. The following inequality holds:

$$
\begin{array}{r}
\left|u^{\tau}(\xi, x, z)\right| \leqslant\left|u^{\tau}\left(\frac{\tau}{2}, x, z\right)\right|+2 \cdot \int_{\frac{\tau}{2}}^{\xi}\left(\left|u^{\tau}\left(\theta-\frac{\tau}{2}, x, z\right)\right|\right)^{p} \cdot\left|M^{\tau}\left(\theta, x, z, u^{\tau}\left(\theta-\frac{\tau}{2}, x, z\right)\right)\right|+ \\
+2 \cdot \int_{\frac{\tau}{2}}^{\xi}|f(\theta, x, z)| d \theta, \quad \frac{\tau}{2}<\xi \leqslant t, \quad t \in\left(\frac{\tau}{2}, \tau\right] .
\end{array}
$$

From the notation (12) and the conditions (7)-(9), since $a(t), b(t) \in C^{1}([0, T])$, it follows that the functions $A_{0}(t), A_{i}(t, x), B_{i}(t, z) i=1,2,3$, and their derivatives with respect to $x$ and $z$ of order up to 6 , including 6 th, are bounded and continuous in $G_{[0, T]}$. Applying first $\sup _{(x, z) \in \mathbb{R}^{2}}$ and then $\sup _{\frac{\tau}{2}<\xi \leqslant t}$ to both parts of the inequality (23) we obtain the estimate

$$
\begin{aligned}
& \sup _{\frac{\tau}{2}<\xi \leqslant t} \sup _{(x, z) \in \mathbb{R}^{2}}\left|u^{\tau}(\xi, x, z)\right| \leqslant \sup _{(x, z) \in \mathbb{R}^{2}}\left|u^{\tau}\left(\frac{\tau}{2}, x, z\right)\right|+2 \cdot \int_{\frac{\tau}{2}}^{\xi} \sup _{\frac{\tau}{2}<\xi \leqslant \theta} \sup _{(x, z) \in \mathbb{R}^{2}}|f(\xi, x, z)| d \theta+ \\
& +2 \cdot \int_{\frac{\tau}{2}}^{\xi}\left(\sup _{\frac{\tau}{2}<\xi \leqslant \theta} \sup _{(x, z) \in \mathbb{R}^{2}}\left|u^{\tau}\left(\xi-\frac{\tau}{2}, x, z\right)\right|\right)^{p} \cdot \sup _{\frac{\tau}{2}<\xi \leqslant \theta} \sup _{(x, z) \in \mathbb{R}^{2}}\left|M^{\tau}\left(\xi, x, z, u^{\tau}\left(\xi-\frac{\tau}{2}, x, z\right)\right)\right| d \theta \\
& \frac{\tau}{2}<\xi \leqslant t, t \in\left(\frac{\tau}{2}, \tau\right] .
\end{aligned}
$$

Taking into account notation (17)-(19), conditions (7)-(9) and the fact that $a(t), b(t) \in$ $C^{1}([0, T])$, from $(24)$ we find

$$
\begin{aligned}
U_{0,0}^{\tau}(t) \leqslant U_{0,0}(0) & +C \cdot \int_{\frac{\tau}{2}}^{t} d \theta+C \cdot \int_{\frac{\tau}{2}}^{t}\left(U_{0,0}^{\tau}\left(\theta-\frac{\tau}{2}\right)\right)^{p} \times \\
& \times\left(1+U_{0,2}^{\tau}\left(\theta-\frac{\tau}{2}\right)+U_{0,1}^{\tau}\left(\theta-\frac{\tau}{2}\right)\right) \cdot\left(1+U_{2,0}^{\tau}\left(\theta-\frac{\tau}{2}\right)+U_{1,0}^{\tau}\left(\theta-\frac{\tau}{2}\right)\right) d \theta .
\end{aligned}
$$

Hereinafter, $C$ denotes (generally speaking, various) constants depending on degree $p$, constants $\delta_{1}, \delta_{2}, \delta_{3}$ from (8), (9) and the constant from (7) which bounds the input data, and independent of the parameter $\tau$. For the sake of convenience, we assume that $C \geqslant 1$.

Let us denote the result of differentiating of function $\left(u^{\tau}(t, x, z)\right)^{p} k_{1}$ times with respect to $x$ 
and $k_{2}$ times with respect to $z$ by $\Delta_{k_{1}, k_{2}}^{\tau}(t, x, z), k_{1}, k_{2}=0,1, \ldots, 6$, that is

$$
\begin{aligned}
& \Delta_{0,0}^{\tau}\left(t-\frac{\tau}{2}, x, z\right)=\left(u^{\tau}\left(t-\frac{\tau}{2}, x, z\right)\right)^{p}, \\
& \Delta_{1,0}^{\tau}\left(t-\frac{\tau}{2}, x, z\right)=p \cdot\left(u^{\tau}\left(t-\frac{\tau}{2}, x, z\right)\right)^{p-1} \cdot \frac{\partial}{\partial x} u^{\tau}\left(t-\frac{\tau}{2}, x, z\right), \\
& \Delta_{0,1}^{\tau}\left(t-\frac{\tau}{2}, x, z\right)=p \cdot\left(u^{\tau}\left(t-\frac{\tau}{2}, x, z\right)\right)^{p-1} \cdot \frac{\partial}{\partial z} u^{\tau}\left(t-\frac{\tau}{2}, x, z\right), \\
& \Delta_{1,1}^{\tau}\left(t-\frac{\tau}{2}, x, z\right)=p \cdot(p-1) \cdot\left(u^{\tau}\left(t-\frac{\tau}{2}, x, z\right)\right)^{p-2} \cdot \frac{\partial}{\partial x} u^{\tau}\left(t-\frac{\tau}{2}, x, z\right) \cdot \frac{\partial}{\partial z} u^{\tau}\left(t-\frac{\tau}{2}, x, z\right)+ \\
& +p \cdot\left(u^{\tau}\left(t-\frac{\tau}{2}, x, z\right)\right)^{p-1} \cdot \frac{\partial^{2}}{\partial x \partial z} u^{\tau}\left(t-\frac{\tau}{2}, x, z\right),
\end{aligned}
$$

and so forth to $\Delta_{6,6}^{\tau}\left(t-\frac{\tau}{2}, x, z\right)$.

According to (18), (19) for $(t, x, z) \in G_{[0, T]}$ the following inequality is valid:

$$
\left|\Delta_{k_{1}, k_{2}}^{\tau}(t, x, z)\right| \leqslant p^{k_{1}+k_{2}} \cdot\left(U^{\tau}(t)\right)^{p}, k_{1}, k_{2}=0,1, \ldots, 6 .
$$

We differentiate equation (15) with respect to $x$ and $z$ from 1 to 6 times and integrate the obtained expression with respect to the time variable. Using the notation (17) and the inequality (26) we apply first $\sup _{(x, z) \in \mathbb{R}^{2}}$ and then $\sup _{\frac{\tau}{2}<\xi \leqslant t}$ to both parts of the obtained inequality. In view of the conditions $(7)-(9)$, notations $(18),(19)$ and the fact that $a(t), b(t) \in C^{1}([0, T])$, we get the estimate

$$
\begin{array}{r}
U_{k_{1}, k_{2}}^{\tau}(t) \leqslant U_{k_{1}, k_{2}}(0)+C \cdot p^{k_{1}+k_{2}} \cdot \int_{\frac{\tau}{2}}^{t}\left(\left(U^{\tau}\left(\theta-\frac{\tau}{2}\right)\right)^{p}+1\right) d \theta+C \cdot p^{k_{1}+k_{2}} \cdot \int_{\frac{\tau}{2}}^{t}\left(U^{\tau}\left(\theta-\frac{\tau}{2}\right)\right)^{p} \times \\
\times \sum_{k_{1}, k_{2}=0}^{6} U_{k_{1}, k_{2}}^{\tau}\left(\theta-\frac{\tau}{2}\right) d \theta+C \cdot p^{k_{1}+k_{2}} \int_{\frac{\tau}{2}}^{t}\left(U^{\tau}\left(\theta-\frac{\tau}{2}\right)\right)^{p} \cdot\left(\sum_{k_{1}, k_{2}=0}^{6} U_{k_{1}, k_{2}}^{\tau}\left(\theta-\frac{\tau}{2}\right)\right)^{2} d \theta \\
k_{1}, k_{2}=0,1, \ldots, 6, \quad t \in\left(\frac{\tau}{2}, \tau\right] . \quad(27)
\end{array}
$$

Summing up the inequalities (25) and (27) and using the estimate (22) we have

$$
U^{\tau}(t) \leqslant U(0)+C \cdot p^{12} \cdot \int_{\frac{\tau}{2}}^{t} \mathcal{P}_{p+2}\left(U^{\tau}\left(\theta-\frac{\tau}{2}\right)\right) d \theta,
$$

where $\mathcal{P}_{p+2}(\chi)=\widetilde{C} \cdot\left(\chi^{p+2}+\chi^{p+1}+\chi^{p}+\ldots+\chi+1\right)$ is a polynomial of degree $p+2, \widetilde{C}$ is a nonzero constant independent of $\tau$.

The latter inequality holds for all $t \in\left(\frac{\tau}{2}, \tau\right]$. Therefore taking into account the properties of definite integrals and the function $U^{\tau}(t)$ we find

$$
U^{\tau}(t) \leqslant U(0)+C \cdot p^{12} \cdot \int_{0}^{\tau} \mathcal{P}_{p+2}\left(U^{\tau}(\theta)\right) d \theta, \quad t \in(0, \tau] .
$$

We consider the Cauchy problem for the ordinary differential equation

$$
\frac{d \omega(t)}{d t}=\mathcal{P}_{p+2}(\omega(t)), \quad \omega(0)=U(0)
$$

By the Cauchy theorem [13], a solution $\omega(t)$ of the problem (29) exists on some interval $\left[0, t_{*}\right]$, where $t_{*} \in(0, T]$ depends on constant $C$ and the initial data $U(0)$. The function $\omega(t)$ increases monotonously on the interval $\left[0, t_{*}\right]$ and $\omega(t) \in C^{1}\left(\left[0, t_{*}\right]\right)$. 
By $(28),(29)$, if $U^{\tau}\left(t_{0}\right) \leqslant \omega\left(t_{0}\right)$ for some $0<t_{0} \leqslant t$, then $U^{\tau}(t) \leqslant \omega(t), t \in\left[t_{0}, t_{*}\right]$. Since $U^{\tau}(0) \leqslant \omega(0)$, the fact that $\omega(t)$ is monotone implies $U^{\tau}(t) \leqslant \omega(\tau), t \in[0, \tau]$.

We consider the first complete time step, where $t \in(\tau, 2 \tau]$. Arguing in the same way as at the zeroth complete step we obtain $U^{\tau}(t) \leqslant \omega(2 \tau), t \in[0,2 \tau]$. After a finite number of steps we have

$$
U^{\tau}(t) \leqslant \omega\left(t_{*}\right) \leqslant C, \quad t \in\left[0, t_{*}\right]
$$

Thus, we have proved the following uniform by $\tau$ estimates:

$$
\left|\frac{\partial^{k_{1}}}{\partial x^{k_{1}}} \frac{\partial^{k_{2}}}{\partial z^{k_{2}}} u^{\tau}(t, x, z)\right| \leqslant C, \quad k_{1}, k_{2}=0,1, \ldots, 6, \quad(t, x, z) \in G_{\left[0, t_{*}\right]} .
$$

Estimates (30) imply that the right parts of equations (14), (15) are bounded uniformly by $\tau$ on any time step. Therefore the left parts of the equations are bounded uniformly by $\tau$ too:

$$
\left|u_{t}^{\tau}(t, x, z)\right| \leqslant C, \quad(t, x, z) \in G_{\left[0, t_{*}\right]} .
$$

Differentiating equations (14), (15) with respect to $x$ and $z$, by (30) we get

$$
\left|\frac{\partial^{k_{1}}}{\partial x^{k_{1}}} \frac{\partial^{k_{2}}}{\partial z^{k_{2}}} u_{t}^{\tau}(t, x, z)\right| \leqslant C, \quad k_{1}, k_{2}=0,1, \ldots, 4, \quad(t, x, z) \in G_{\left[0, t_{*}\right]} .
$$

Estimates (30), (31) guarantee that the assumptions of Arzela's compactness theorem are satisfied. By this theorem, some subsequence $u^{\tau_{k}}(t, x, z)$ of the sequence of solutions $u^{\tau}(t, x, z)$ to the problem (14)-(16) with corresponding derivatives converges to a function $u(t, x, z) \in C_{t, x, z}^{0,4,4}\left(G_{\left[0, t_{*}\right]}^{M}\right)$. In view of the theorem about convergence of the weak approximation method, the function $u(t, x, z)$ is a solution of the problem (13), (3), moreover $u(t, x, z) \in C_{t, x, z}^{1,4,4}\left(G_{\left[0, t_{*}\right]}^{M}\right)$, where $M>0$ is an integer constant,

$$
\begin{gathered}
G_{\left[0, t_{*}\right]}^{M}=\left\{(t, x, z)\left|0 \leqslant t \leqslant t_{*},\right| x|\leqslant M,| z \mid \leqslant M\right\}, \\
C_{t, x, z}^{l, l_{1}, l_{2}}\left(G_{\left[0, t_{*}\right]}^{M}\right)=\left\{u(t, x, z) \mid \frac{\partial^{k}}{\partial t^{k}} u, \frac{\partial^{k_{1}+k_{2}}}{\partial x^{k_{1}} \partial z^{k_{2}}} u \in C\left(G_{\left[0, t_{*}\right]}^{M}\right),\right. \\
\left.k=0,1, \ldots, l, \quad k_{1}=0,1, \ldots, l_{1}, \quad k_{2}=0,1, \ldots, l_{2}\right\} .
\end{gathered}
$$

We choose constant $M$ arbitrarily, therefore the solution $u(t, x, z)$ of the problem (13), (3) belongs to the class $C_{t, x, z}^{1,4,4}\left(G_{\left[0, t_{*}\right]}\right)$.

Furthermore the following estimations are valid for $(t, x, z) \in G_{\left[0, t_{*}\right]}$ :

$$
\left|\frac{\partial^{k_{1}}}{\partial x^{k_{1}}} \frac{\partial^{k_{2}}}{\partial z^{k_{2}}} u(t, x, z)\right| \leqslant C, \quad k_{1}, k_{2}=0,1, \ldots, 4 .
$$

From continuous differentiability of $a(t), b(t)$, the conditions (7)-(9) and the estimation (32), from (10) and (13) it follows that $u(t, x, z), \lambda(t, x, z)$ belong to the class

$$
Z\left(t_{*}\right)=\left\{u(t, x, z), \lambda(t, x, z) \mid u \in C_{t, x, z}^{1,4,4}\left(G_{\left[0, t_{*}\right]}\right), \lambda(t, x, z) \in C_{t, x, z}^{0,2,2}\left(G_{\left[0, t_{*}\right]}\right)\right\},
$$

and satisfy the following inequality:

$$
\sum_{k_{1}, k_{2}=0}^{4}\left|\frac{\partial^{k_{1}}}{\partial x^{k_{1}}} \frac{\partial^{k_{2}}}{\partial z^{k_{2}}} u(t, x, z)\right|+\sum_{k_{1}, k_{2}=0}^{2}\left|\frac{\partial^{k_{1}}}{\partial x^{k_{1}}} \frac{\partial^{k_{2}}}{\partial z^{k_{2}}} \lambda(t, x, z)\right| \leqslant C .
$$




\section{Existence of solution of the inverse problem}

Now we prove that the pair of functions $u(t, x, z), \lambda(t, x, z)$, where $\lambda(t, x, z)$ is defined by (10) and satisfies the condition (4), is a solution of the inverse problem (1)-(5). Since $u(t, x, z)$ is a solution of the direct problem (13), (3), substituting $u(t, x, z), \lambda(t, x, z)$ into (1), (3) we obtain the identities.

Let us prove that the overdetermination conditions (5) are fulfilled.

In the strip $\mathrm{P}_{[0, T]}=\{(t, y) \mid 0 \leqslant t \leqslant T, y \in \mathbb{R}\}$ we consider the auxiliary Cauchy problem for the equation

$$
\begin{aligned}
v_{t}(t, y)=a_{1}(t) \cdot v_{y y}(t, y)+a_{2}(t) \cdot v_{y}(t, y)+ & a_{3}(t, y) \cdot v(t, y)- \\
& -a_{4}(t, y) \cdot v_{y y}(t, d(t))-a_{5}(t, y) \cdot v_{y}(t, d(t)),
\end{aligned}
$$

with the initial condition

$$
v(0, y)=r(y)
$$

Suppose that the functions $a_{1}(t), a_{2}(t), a_{3}(t, y), a_{4}(t, y), a_{5}(t, y), r(y)$ and all their derivatives are continuous and bounded in $\mathrm{P}_{[0, T]}, d(t)$ is a smooth bounded curve.

The following statement takes place.

Lemma 1. If solution $v(t, y) \in C_{t, y}^{1,4}\left(\mathrm{P}_{[0, T]}\right)$ of the problem (34), (35) exists and satisfies the condition

$$
\sum_{k=0}^{4}\left|\frac{\partial^{k}}{\partial y^{k}} v(t, y)\right| \leqslant C
$$

then it is unique. Here,

$$
C_{t, y}^{1,4}\left(\mathrm{P}_{[0, T]}\right)=\left\{v(t, y) \mid v_{t}(t, y), \frac{\partial^{k}}{\partial y^{k}} v(t, y) \in C\left(\mathrm{P}_{[0, T]}\right), k=0,1, \ldots, 4\right\}
$$

The proof of the Lemma 1 basically repeats the proof of Lemma in paper [7].

We substitute $z=a(t)$ into (13). Transforming the obtained relation and taking into consideration that

$$
\begin{aligned}
& \frac{(u(t, x, a(t)))^{p}}{(\varphi(t, x))^{p}}=\frac{(u(t, x, a(t)))^{p}-(\varphi(t, x))^{p}+(\varphi(t, x))^{p}}{(\varphi(t, x))^{p}}= \\
& \quad=(u(t, x, a(t))-\varphi(t, x)) \cdot \frac{u^{p-1}+u^{p-2} \cdot \varphi+u^{p-3} \cdot \varphi^{2}+\ldots+u \cdot \varphi^{p-2}+\varphi^{p-1}}{(\varphi(t, x))^{p}}+1
\end{aligned}
$$

we find

$$
\begin{gathered}
u_{t}(t, x, a(t))+u_{z}(t, x, a(t)) \cdot a^{\prime}(t)-\varphi_{t}(t, x)=\alpha_{1}(t) \cdot\left(u_{x x}(t, x, a(t))-\varphi_{x x}(t, x)\right)+ \\
+\beta_{1}(t) \cdot\left(u_{x}(t, x, a(t))-\varphi_{x}(t, x)\right)+D_{1}(t, x) \cdot(u(t, x, a(t))-\varphi(t, x))- \\
-D_{2}(t, x) \cdot \alpha_{1}(t) \cdot\left(u_{x x}(t, b(t), a(t))-\varphi_{x x}(t, b(t))\right)+D_{3}(t, x) \cdot \beta_{1}(t) \cdot\left(u_{x}(t, b(t), a(t))-\varphi_{x}(t, b(t))\right),
\end{gathered}
$$


where

$$
\begin{aligned}
& D_{0}(t)=\psi_{t}(t, a(t))-\alpha_{2}(t) \cdot \psi_{z z}(t, a(t))-\beta_{2}(t) \cdot \psi_{z}(t, a(t))-f(t, b(t), a(t))- \\
& -\alpha_{1}(t) \cdot u_{x x}(t, b(t), a(t))-\beta_{1}(t) \cdot u_{x}(t, b(t), a(t)) \cdot\left(1+b^{\prime}(t)\right) ; \\
& D_{1}(t, x)=\frac{1}{D_{0}(t)} \cdot \frac{u^{p-1}+u^{p-2} \cdot \varphi+u^{p-3} \cdot \varphi^{2}+\ldots+u \cdot \varphi^{p-2}+\varphi^{p-1}}{(\varphi(t, x))^{p}} \times \\
& \times\left(\left(\varphi_{t}(t, x)-\alpha_{1}(t) \cdot \varphi_{x x}(t, x)-\beta_{1}(t) \cdot \varphi_{x}(t, x)-f(t, x, a(t))\right) \times\right. \\
& \times\left(\psi_{t}(t, a(t))-\alpha_{2}(t) \cdot \psi_{z z}(t, a(t))-\beta_{2}(t) \cdot \psi_{z}(t, a(t))-f(t, b(t), a(t))\right)+ \\
& +\left(\varphi_{t}(t, x)-\alpha_{1}(t) \cdot \varphi_{x x}(t, x)-\beta_{1}(t) \cdot \varphi_{x}(t, x)-f(t, x, a(t))\right) \cdot\left(-\alpha_{1}(t) \cdot u_{x x}(t, b(t), a(t))-\right. \\
& \left.-\beta_{1}(t) \cdot u_{x}(t, b(t), a(t)) \cdot\left(1+b^{\prime}(t)\right)\right)+\left(\psi_{t}(t, a(t))-\alpha_{2}(t) \cdot \psi_{z z}(t, a(t))-\beta_{2}(t) \cdot \psi_{z}(t, a(t))-\right. \\
& -f(t, b(t), a(t))) \cdot\left(-\alpha_{2}(t) \cdot u_{z z}(t, x, a(t))-\beta_{2}(t) \cdot u_{z}(t, x, a(t)) \cdot\left(1+a^{\prime}(t)\right)\right)+ \\
& +\left(-\alpha_{1}(t) \cdot u_{x x}(t, b(t), a(t))-\beta_{1}(t) \cdot u_{x}(t, b(t), a(t)) \cdot\left(1+b^{\prime}(t)\right)\right) \times \\
& \left.\times\left(-\alpha_{2}(t) \cdot u_{z z}(t, x, a(t))-\beta_{2}(t) \cdot u_{z}(t, x, a(t)) \cdot\left(1+a^{\prime}(t)\right)\right)\right) ; \\
& D_{2}(t, x)=\frac{u_{t}(t, x, a(t))-\alpha_{1}(t) \cdot u_{x x}(t, x, a(t))-\beta_{1}(t) \cdot u_{x}(t, x, a(t))-\alpha_{2}(t) \cdot u_{z z}(t, x, a(t))}{D_{0}(t)}- \\
& -\frac{\beta_{2}(t) \cdot u_{z}(t, x, a(t))+f(t, x, a(t))}{D_{0}(t)} \\
& D_{3}(t, x)=D_{2}(t, x) \cdot\left(1+b^{\prime}(t)\right) \text {. }
\end{aligned}
$$

We introduce the notation: $\nu^{1}(t, x)=u(t, x, a(t))-\varphi(t, x)$. Hence,

$$
\nu_{t}^{1}(t, x)=u_{t}(t, x, a(t))+u_{z}(t, x, a(t)) \cdot a^{\prime}(t)-\varphi_{t}(t, x)
$$

From the compatibility conditions (6) it follows that

$$
\nu^{1}(0, x)=u(0, x, a(0))-\varphi(0, x)=u_{0}(x, a(0))-\varphi(0, x)=0 .
$$

Consequently, the function $\nu^{1}(t, x)$ is a solution of the following problem:

$$
\begin{gathered}
\nu_{t}^{1}(t, x)=\alpha_{1}(t) \cdot \nu_{x x}^{1}(t, x)+\beta_{1}(t) \cdot \nu_{x}^{1}(t, x)+D_{1}(t, x) \cdot \nu^{1}(t, x)- \\
-D_{2}(t, x) \cdot \alpha_{1}(t) \cdot \nu_{x x}^{1}(t, b(t))-D_{3}(t, x) \cdot \beta_{1}(t) \cdot \nu_{x}^{1}(t, b(t)), \\
\nu^{1}(0, x)=0 .
\end{gathered}
$$

In accordance with the constraints (7)-(9), the inequality (33) and the fact that $a(t)$, $b(t) \in C^{1}([0, T])$, the functions $D_{1}(t, x), D_{2}(t, x), D_{3}(t, x)$ and their derivatives with respect to $x$ to the second order, including $2 \mathrm{nd}$, are continuous and bounded in $G_{\left[0, t_{*}\right]}$. Therefore, the problem (36), (37) satisfies the assumptions of Lemma 1 (where $a_{1}=\alpha_{1}(t), a_{2}=\beta_{1}(t), a_{3}=D_{1}(t, x)$, $\left.a_{4}=D_{2}(t, x) \cdot \alpha_{1}(t), a_{5}=D_{3}(t, x) \cdot \beta_{1}(t), d=a(t), r=0\right)$. From Lemma 1 it follows that the solution of problem (36), (37) is unique. This solution is the function $\nu^{1}(t, x)=0$. This means that $u(t, x, a(t))=\varphi(t, x)$. Hence the first of the overdetermination conditions (5) holds. Similarly, 
using the compatibility conditions (6) we prove that the second of overdetermination conditions (5) is valid: $u(t, b(t), z)=\psi(t, z)$.

Similarly, using the compatibility conditions (6) we prove that the second of overdetermination conditions (5) is valid: $u(t, b(t), z)=\psi(t, z)$.

Thus the following theorem has been proved.

Theorem. Let the conditions (6)-(9) be satisfied. Then there exists a constant $0<t_{*} \leqslant T$ depending on the degree $p$ and on the constants $\delta_{1}, \delta_{2}, \delta_{3}, C$ from (7)-(9) and such that a solution $u(t, x, z), \lambda(t, x, z)=\lambda_{1}(t, x) \cdot \lambda_{2}(t, z)$ of the inverse problem (1)-(5) in the class $Z\left(t_{*}\right)$ exists and satisfies (33).

\section{Example}

For the problem under study there is example of the initial data satisfying the assumptions of the theorem and the corresponding solution.

In the strip $G_{[0, T]}=\left\{(t, x, z) \mid 0 \leqslant t \leqslant T,(x, z) \in \mathbb{R}^{2}\right\}$ we consider the Cauchy problem for the parabolic equation

$$
u_{t}=u_{x x}+u_{z z}+u_{x}+u_{z}+u \cdot \lambda(t, x, z)-2 \cos x \cdot(\cos z+A),
$$

$A>1$ is a constant, $t \in(0, T],(x, z) \in \mathbb{R}^{2}$, with the initial condition $u(0, x, z)=u_{0}(x, z)=$ $e^{x}(\sin z+A)$. In addition to the function $u(t, x, z)$ it is required to find the function $\lambda(t, x, z)=$ $\lambda_{1}(t, x) \cdot \lambda_{2}(t, z)$.

Let $a(t)=t, b(t)=t+2 \in C^{1}([0, T])$. Then the overdetermination conditions have the form:

$$
u(t, x, a(t))=\varphi(t, x)=e^{t+x} \cdot(\sin t+A), \quad u(t, b(t), z)=\psi(t, z)=e^{2 t+2} \cdot(\sin z+A) .
$$

The compatibility conditions hold

$$
\begin{gathered}
u_{0}(x, a(0))=\varphi(0, x)=A \cdot e^{x}, \quad u_{0}(b(0), z)=\psi(0, z)=e^{2} \cdot(\sin z+A), \\
\varphi(t, b(t))=\psi(t, a(t))=e^{2 t+2} \cdot(\sin t+A) .
\end{gathered}
$$

The functions $f(t, x, z)=-2 \cos x \cdot(\cos z+A), u_{0}(x, z), \varphi(t, x), \psi(t, z)$ satisfy the smoothness and boundedness conditions $(7)$.

We verify fulfilment of the conditions (8):

$$
\begin{gathered}
|\varphi(t, x)|=\left|e^{t+x}\right| \cdot|\sin t+A| \geqslant e^{x} \cdot(A-1) \geqslant \varepsilon \cdot(A-1)>0, \quad 0<\varepsilon \ll 1, \quad \forall t \in[0, T], \quad \forall x \in \mathbb{R}, \\
|\psi(t, z)|=\left|e^{2 t+2}\right| \cdot|\sin z+A| \geqslant e^{2} \cdot(A-1)>0, \quad \forall t \in[0, T], \quad \forall z \in \mathbb{R} .
\end{gathered}
$$

After that we consider (9):

$$
\begin{gathered}
\mid e^{2 t+2}(\sin t+\cos t+A)-e^{2 t+2}(\sin t+A)+e^{2 t+2} \sin t-e^{2 t+2}(\sin t+A)-2 e^{2 t+2} \cos t+ \\
+2 \cos (t+2)(\cos t+A)|\geqslant|(A+\cos t) \cdot\left(e^{2 t+2}-2 \cos (t+2)\right)|\geqslant(A-1) \cdot| e^{2 t+2}-2 \cos (t+2) \mid \geqslant \\
\geqslant(A-1) \cdot\left(\left|e^{2 t+2}\right|-2|\cos (t+2)|\right) \geqslant(A-1) \cdot\left(e^{2}-2\right)>0, \quad \forall t \in[0, T] .
\end{gathered}
$$

Consequently the conditions (8), (9) are satisfied for $\delta_{1}=\varepsilon \cdot(A-1), \delta_{2}=e^{2} \cdot(A-1)$, $\delta_{3}=(A-1) \cdot\left(e^{2}-2\right)$. 
Then we can find the functions $u(t, x, z), \lambda(t, x, z)$ :

$$
\begin{gathered}
u(t, x, z)=e^{t+x} \cdot(\sin z+A) \\
\lambda(t, x, z)=\lambda_{1}(t, x) \cdot \lambda_{2}(t, z)=-\left(1-\frac{2 \cos x}{e^{t+x}}\right) \cdot \frac{\cos z+A}{\sin z+A} .
\end{gathered}
$$

Indeed, the obtained solution satisfies equation (1):

$$
\begin{gathered}
e^{t+x} \cdot(\sin z+A)=e^{t+x} \cdot(\sin z+A)-e^{t+x} \cdot \sin z+e^{t+x} \cdot(\sin z+A)+e^{t+x} \cdot \cos z- \\
-e^{t+x} \cdot(\sin z+A) \cdot\left(1-\frac{2 \cos x}{e^{t+x}}\right) \cdot \frac{\cos z+A}{\sin z+A}-2 \cos x \cdot(\cos z+A), \\
e^{t+x} \cdot A+e^{t+x} \cos z-\left(e^{t+x}-2 \cos x\right) \cdot(\cos z+A)-2 \cos x \cdot(\cos z+A)=0, \\
(\cos z+A) \cdot\left(e^{t+x}-e^{t+x}+2 \cos x-2 \cos x\right)=0, \\
0=0 .
\end{gathered}
$$

This example shows that the set of solutions to the problem under study is not empty.

The research for this paper was carried out in Siberian Federal University within the framework of the project «Multidimensional Complex Analysis and Differential Equations» funded by the grant of the Russian Federation Government to support scientific research under supervision of a leading scientist, no. 14.Y26.31.0006.

\section{References}

[1] N.N.Yanenko, The Method of Fractional Steps for Solving Multi-Dimensional Problems of Mathematical Physics, Novosibirsk, Nauka, 1967 (in Russian).

[2] Yu.Ya.Belov, Inverse Problems for Partial Differential Equations, Utrecht, VSP, 2002.

[3] Yu.Ya.Belov, On Estimates of Solutions of the Split Problems for Some Multi-Dimensional Partial Differential Equations, Journal of Siberian Federal University. Mathematics \& Physics, 2(2009), no. 3, 258-270.

[4] Yu.Ya.Belov, I.V.Frolenkov, Coefficient Identification Problems for Semilinear Parabolic Equations, Doklady Mathematics, 72(2005), no. 2, 737-739.

[5] E.N.Kriger, I.V.Frolenkov, An Identification Problem of the Special Form Coefficient at a Nonlinear Term for a Two-Dimensional Parabolic Equation, Differential equations. Function spaces. Approximation theory, International Conference dedicated to the 105th anniversary of the birthday of Sergei L. Sobolev (August 18 - 24, 2013, Novosibirsk, Russia), Abstracts, Novosibirsk, Sobolev Institute of Mathematics, 2013, 176 (in Russian).

[6] Yu.Ya.Belov, I.V.Frolenkov, On the Problem of Identification of Two Coefficients of a Parabolic Semi-Linear Overdetermined Equation Defined on a Smooth Curve, Special issue of journal Kompyuternye tehnologii devoted to N.N. Yanenko's 85-th anniversary, 11(2006), no. 1, 46-54 (in Russian). 
[7] E.N.Kriger, I.V.Frolenkov, An Identification Problem of Coefficient for Two-Dimensional Semilinear Parabolic Equation with the Cauchy Data, Russian Mathematics, 59(2015), no. 5, $17-31$.

[8] E.G.Savateev, On the Problem of Identification of a Coefficient in a Parabolic Equation, Siberskii Mat. Zhurnal, 36(1995), no. 1, 177-185 (in Russian).

[9] A.I.Kozhanov, Nonlinear Loaded Equations and Inverse Problems, Vych. Matematika $i$ Mat. Physika, 44(2004), no. 4, 694-716 (in Russian).

[10] I.V.Frolenkov, E.N.Kriger, Existence of a Solution of the Problem of Identification of a Coefficient at the Source Function, Journal of Mathematical Sciences, 203(2014), no. 4, $464-477$.

[11] I.V.Frolenkov, E.N.Kriger, An Identification Problem of the Source Function of the Special Form in Two-Dimensional Parabolic Equation, Journal of Siberian Federal University. Mathematics \& Physics, 3(2010), no. 4, 556-564 (in Russian).

[12] I.V.Frolenkov, E.N.Kriger, An Identification Problem of Coefficient in the Special Form at Source Function for Multi-Dimensional Parabolic Equation with Cauchy Data, Journal of Siberian Federal University. Mathematics \& Physics, 6(2013), no. 2, 186-199.

[13] L.S. Pontryagin, Ordinary Differential Equations, Moscow, Nauka, 1982 (in Russian).

\section{Об одной задаче идентификации коэффициента специального вида при нелинейном младшем члене в двумерном полулинейном параболическом уравнении}

\section{Екатерина Н. Кригер}

Игорь В. Фроленков

Институт математики и фундаментальной информатики Сибирский федеральный университет Свободный, 79, Красноярск, 660041

Россия

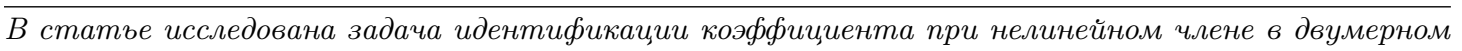
полулинейном параболическом уравнении с данными Коши и условиями переопределения, заданными на гладкой кривой. В предположении, что искомый коэффициент представим в виде произведения двух функций, каждая из которых зависит от временной и только одной пространственной переменной, доказана разрешимость рассмотренной задачи «в малом» в классе гладких ограниченных функций. Построен пример входных данных, удовлетворяющих условиям доказанной теоремы, и соответствующего им решения.

Ключевые слова: обратная задача, полулинейное параболическое уравнение, задача Коши, коэффичиент при младием члене, метод слабой аппроксимации, локальная разрешимость, условия переопределения на гладкой кривой. 